University of Wollongong

Research Online

Faculty of Engineering and Information

Faculty of Engineering and Information

Sciences - Papers: Part A

Sciences

$1-1-2014$

Improving the accuracy performance of phase-shifting profilometry for the measurement of objects in motion

Lei Lu

University of Wollongong, II490@uowmail.edu.au

Jiangtao Xi

University of Wollongong, jiangtao@uow.edu.au

Yanguang Yu

University of Wollongong, yanguang@uow.edu.au

Qinghua Guo

University of Wollongong, qguo@uow.edu.au

Follow this and additional works at: https://ro.uow.edu.au/eispapers

Part of the Engineering Commons, and the Science and Technology Studies Commons

Research Online is the open access institutional repository for the University of Wollongong. For further information contact the UOW Library: research-pubs@uow.edu.au 


\title{
Improving the accuracy performance of phase-shifting profilometry for the measurement of objects in motion
}

\author{
Abstract \\ This Letter presents a new approach to reducing the errors associated with the shape measurement of a \\ rigid object in motion by means of phase-shifting profilometry. While the work previously reported is only \\ valid for the case of two-dimensional (2-D) movement, the proposed method is effective for a situation in \\ which the object moves in a three-dimensional (3-D) space. First, a new model is proposed to describe the \\ fringe patterns reflected from the object, which is subject to 3-D movement. Then, an iterative least- \\ squares algorithm is presented to estimate the phase map. Experiments show that, in contrast to \\ conventional phase-shifting profilometry, the proposed method is capable of significantly reducing the \\ error caused by the 3-D movement of the object.

\section{Disciplines} \\ Engineering | Science and Technology Studies

\section{Publication Details} \\ L. Lu, J. Xi, Y. Yu \& Q. Guo, "Improving the accuracy performance of phase-shifting profilometry for the \\ measurement of objects in motion," Optics Letters, vol. 39, (23) pp. 6715-6718, 2014.
}




\title{
Improving the accuracy performance of phase shifting profilometry for the measurement of objects in motion
}

\author{
Lei Lu, ${ }^{1}$ Jiangtao $\mathrm{Xi}^{1}{ }^{1, *}$ Yanguang Yu, ${ }^{1}$ and Qinghua Guo ${ }^{1}$ \\ ${ }^{1}$ School of Electrical Computer and Telecommunications Engineering, University of Wollongong, Wollongong, NSW, 2522, \\ Australia \\ *Corresponding author: jiangtao@uow.edu.au
}

Received July 29, 2014; revised October 5, 2014; accepted October 10, 2014;

posted October 14, 2014 (Doc. ID 220038); published November 26, 2014

\begin{abstract}
This letter presents a new approach to reduce the errors associated with the shape measurement of a rigid object in motion by means of phase shifting profilometry. While the work previously reported is only valid for the case of two-dimensional (2D) movement, the proposed method is effective for the situation where the object moves in three-dimensional (3-D) space. Firstly, a new model is proposed to describe the fringe patterns reflected from the object which is subject to a 3-D movement. Then, an iterative least-squares algorithm is presented to estimate the phase map. Experiments show that, in contrast to conventional phase shifting profilometry, the proposed method is capable of significantly reducing the error caused by the 3D movement of the object. (c) 2010 Optical Society of America

OCIS Codes: $120.6650,120.5050,100.2650,050.5058$
\end{abstract}

Phase shifting profilometry (PSP) is one of the most promising approaches for non-destructive 3 -D shape measurement. However, when the multiple fringe patterns are projected, the object must be kept static. Measurement errors will occur if the object moves.

The above problem can be addressed from a number of aspects. Zhang, et al., proposed a modified two-plus-one phase shifting algorithm to address the problem by projection of two sinusoidal fringe patterns and a uniform flat image [1]. As the height information is only contained in the two sinusoidal fringe patterns, the error due to motion is smaller than the traditional PSP. However, the error still occurs when the object moves during the projections of the two sinusoidal fringe patterns. Wang, et al., proposed to use a high speed camera of 5,000 frames per second to measure the moving object [2]. The object can be considered as static during the projection of the phase shifted fringe patterns. However, use of such high speed equipment implies significant increase in the cost of the system implementation. Based on analysis of the influence on the fringe patterns caused by the movement, $\mathrm{Lu}$, et al., proposed a method to reduce the error caused by the rigid object movement [3]. However, the method presented in Ref. [3] is only applicable to the cases of 2-D movement of the object, which is obviously limited in terms of its practical applications.

In this letter, a new method is proposed to cope with the errors caused by the 3-D movement of the object in PSP. In particular, we consider the case that the object has a rigid shape, and the movement consists of a translation in the direction of height and a 2-D movement in the plane perpendicular to the direction of height. The details of the proposed algorithm are described below.

Let us work out the relationship between the 3-D movement of the object and the fringe patterns. If the object is kept static and N-step PSP is used, the fringe patterns acquired from the reference plane and the object can be expressed as:

$$
\left\{\begin{array}{l}
s_{n}(x, y)=a+b \cos [\omega(x, y)+2 \pi n / N] \\
d_{n}(x, y)=a+b \cos [\omega(x, y)+\Phi(x, y)+2 \pi n / N]
\end{array}\right.
$$

where $n=1,2, \ldots, N ; s_{n}(x, y)$ and $d_{n}(x, y)$ are the $n$-th fringe patterns on the reference plane and the object respectively; $a$ is the background intensity resulting from pattern offset and ambient light; $b$ is the amplitude of the intensity of the sinusoidal fringe patterns. As we only consider the constant background intensity and objects with diffuse surface, $a$ and $b$ can be seen as constant; $\omega(x, y)$ is the phase value on the reference plane; $\Phi(x, y)$ is the phase difference between the object and the reference plane caused by the shape of the object. When $\Phi(x, y)$ is obtained, the shape of the object can be calculated by:

$$
h(x, y)=\frac{l_{0} \Phi(x, y)}{\Phi(x, y)-2 \pi f_{0} d_{0}}
$$

where $h(x, y)$ is the height distribution of object; $l_{0}$ is the distance between the camera and the reference plane; $f_{0}$ is the spatial frequency of the fringe patterns; $d_{0}$ is the distance between the camera and the projector.

Now let us consider the case where the object is subject to a 3-D movement. Because of the movement, the height distribution changes from $h(x, y)$ to $\tilde{h}(x, y)$. Due to the rigid nature of the subject shape, a point $(x, y)$ on the object is moved to $(u, v)$ following the relationship below:

$$
\left[\begin{array}{c}
x \\
y \\
h(x, y)
\end{array}\right]=\mathbf{R}\left[\begin{array}{c}
u \\
v \\
\tilde{h}(u, v)
\end{array}\right]+\mathbf{T},\left[\begin{array}{c}
u \\
v \\
\tilde{h}(u, v)
\end{array}\right]=\overline{\mathbf{R}}\left[\begin{array}{c}
x \\
y \\
h(x, y)
\end{array}\right]+\overline{\mathbf{T}} \text {. }
$$

where $\mathbf{R}, \overline{\mathbf{R}}, \mathbf{T}$ and $\overline{\mathbf{T}}$ are referred to as rotation matrixes and translation vectors. As we only consider the case that the movement is the 
combination of a translation in the direction of height and a 2-D movement in the plane perpendicular to the direction of height, we have:

$\mathbf{R}=\left[\begin{array}{ccc}r_{11} & r_{12} & 0 \\ r_{21} & r_{22} & 0 \\ 0 & 0 & 1\end{array}\right], \quad \mathbf{T}=\left[\begin{array}{c}t_{1} \\ t_{2} \\ t_{3}\end{array}\right], \quad \overline{\mathbf{R}}=\left[\begin{array}{ccc}\bar{r}_{11} & \bar{r}_{12} & 0 \\ \bar{r}_{21} & \bar{r}_{22} & 0 \\ 0 & 0 & 1\end{array}\right], \quad \overline{\mathbf{T}}=\left[\begin{array}{c}\overline{t_{1}} \\ \overline{t_{2}} \\ \overline{t_{3}}\end{array}\right]$.

Because the movement in the direction of height is exclusively a translation, we have:

$$
\tilde{h}^{x-y}(u, v)=h^{x-y}(x, y)+\bar{t}_{3}=h^{x-y}[f(u, v), g(u, v)]+\bar{t}_{3}(5)
$$

where $x-y$ denotes the coordinate system in which the functions are defined and

$$
f(u, v)=r_{11} u+r_{12} v+t_{1}, \quad g(u, v)=r_{21} u+r_{22} v+t_{2} .
$$

Without loss of generality, $(u, v)$ can be replaced by $(x, y)$. Therefore, Eq. (5) yields the following:

$$
\tilde{h}^{x-y}(x, y)=h^{x-y}[f(x, y), g(x, y)]+\bar{t}_{3}
$$

Define the object fringe patterns after movement as:

$$
\tilde{d}_{n}^{x-y}(x, y)=a+b \cos [\omega(x, y)+\tilde{\Phi}(x, y)+2 \pi n / N]
$$

where $\tilde{\Phi}(x, y)$ is the phase difference at point $(x, y)$ after movement. Generally $l_{0}$ is much larger than the measured object and the height variation $\bar{t}_{3}$. The phase variations caused by $\bar{t}_{3}$ for each point of object are approximately same. Because of Eq. (7), we have

$$
\tilde{\Phi}(x, y)=\Phi[f(x, y), g(x, y)]+\Phi^{\prime}
$$

where $\Phi^{\prime}$ is the phase variation which is caused by $\bar{t}_{3}$. Submit Eq. (9) into Eq. (8), we have

$$
\begin{aligned}
& \tilde{d}_{n}^{x-y}(x, y) \\
& =a+b \cos \left\{\omega(x, y)+\Phi[f(x, y), g(x, y)]+\Phi^{\prime}+2 \pi n / N\right\}(10)
\end{aligned}
$$

Note that in order to avoid the phase ambiguity, $\Phi^{\prime}$ is limited to less than $2 \pi$, requiring that the object movement is smaller than a single fringe. Now, let us consider Eq. (10) in a new coordinate system $\xi-\eta$ following the relationship below:

$$
\left[\begin{array}{l}
x \\
y
\end{array}\right]=\left[\begin{array}{ll}
\bar{r}_{11} & \bar{r}_{12} \\
\bar{r}_{21} & \bar{r}_{22}
\end{array}\right]\left[\begin{array}{l}
\xi \\
\eta
\end{array}\right]+\left[\begin{array}{l}
\bar{t}_{1} \\
\overline{t_{2}}
\end{array}\right]
$$

In $\xi-\eta$ coordinate system, Eq. (10) becomes:

$$
\begin{aligned}
& \tilde{d}_{n}^{\xi-\eta}(\xi, \eta)=\tilde{d}_{n}^{x-y}[\bar{f}(\xi, \eta), \bar{g}(\xi, \eta)] \\
& =a+b \cos \left\{\omega[\bar{f}(\xi, \eta), \bar{g}(\xi, \eta)]+\Phi(\xi, \eta)+\Phi^{\prime}+2 \pi n / N\right\}
\end{aligned}
$$

where

$$
\bar{f}(u, v)=\bar{r}_{11} u+\bar{r}_{12} v+\bar{t}_{1}, \quad \bar{g}(u, v)=\bar{r}_{21} u+\bar{r}_{22} v+\bar{t}_{2} .
$$

Use $(x, y)$ to replace $(\xi, \eta)$ and extend Eq. (12) to Nstep PSP, yielding the following:

$$
\begin{aligned}
& \tilde{d}_{n}(x, y)=\tilde{d}_{n}^{x-y}\left[\bar{f}_{n}(x, y), \bar{g}_{n}(x, y)\right] \\
& =a+b \cos \left\{\omega\left[\bar{f}_{n}(x, y), \bar{g}_{n}(x, y)\right]+\Phi(x, y)+\Phi_{n}^{\prime}+2 \pi n / N\right\}
\end{aligned}
$$

Equation (14) describes the influence of the 3-D movement on the fringe patterns. To simplify the expression, Eq. (14) can be rewritten as following:

$$
\tilde{d}_{n}(x, y)=a+b \cos \left[\omega_{n}^{\prime}(x, y)+\Phi(x, y)+\delta_{n}\right]
$$

where $\omega_{n}^{\prime}(x, y)=\omega\left[\bar{f}_{n}(x, y), \bar{g}_{n}(x, y)\right]$ and it can be obtained by the fringe patterns of reference plane and Eq. (13); $\delta_{n}=\Phi_{n}^{\prime}+2 \pi n / N$ is the phase sift amount caused by the phase shift of PSP and height variations $\bar{t}_{3}$ for each step, which is unknown in advance. In order to obtain $\Phi(x, y)$, we must estimate the value of $\delta_{n}$. Inspired by the methods presented in Ref. [4-6] which can extract the random phase shifts for other applications, we propose to employ iterative least-squares approach to obtain $\delta_{n}$ and $\Phi(x, y)$. The proposed method starts from an initial value of $\delta_{n}=2 \pi n / N$ and can be implemented by the following steps.

Step 1: Estimate $\Phi(x, y)$ when an estimation of $\delta_{n}$ is available (it takes the initial value for the first iteration). To this end we rewrite Eq. (15) as:

$$
\begin{aligned}
& \tilde{d}_{n}(x, y)= \\
& a+B(x, y) \cos \left[\omega_{n}^{\prime}(x, y)+\delta_{n}\right]+C(x, y) \sin \left[\omega_{n}^{\prime}(x, y)+\delta_{n}\right](16)
\end{aligned}
$$

where $B(x, y)=b \cos \Phi(x, y)$ and $C(x, y)=-b \sin \Phi(x, y)$. Assuming that there are $M$ pixels in one fringe pattern, and when $\delta_{n}$ is known, there are $2 \mathrm{M}+1$ unknowns and MN equations in Eq. (16). When $N \geq 3$, the unknowns can be obtained by the overdetermined least-squares method. Apply Eq. (14) on the measured fringe patterns $\tilde{d}_{n}^{x-y}$ to obtain $d_{n}^{m}(x, y)$, then, the sum of the squared error in each pixel is:

$$
S(x, y)=\sum_{n=1}^{N}\left[\tilde{d}_{n}(x, y)-d_{n}^{m}(x, y)\right]^{2}
$$

Based on Eq. (17), the least-squares criteria satisfy that $\partial S(x, y) / \partial a=0 \quad, \quad \partial S(x, y) / \partial B(x, y)=0 \quad$ and $\partial S(x, y) / \partial C(x, y)=0$, yield the following:

$$
\mathbf{X}(x, y)=\mathbf{A}^{-1}(x, y) \mathbf{B}(x, y)
$$

where

$$
\begin{aligned}
& \mathbf{A}(x, y)= \\
& {\left[\begin{array}{ccc}
N & \sum_{n=1}^{N} \cos \left[\omega_{n}^{\prime}(x, y)+\delta_{n}\right] & \sum_{n=1}^{N} \sin \left[\omega_{n}^{\prime}(x, y)+\delta_{n}\right] \\
\sum_{n=1}^{N} \cos \left[\omega_{n}^{\prime}(x, y)+\delta_{n}\right] & \sum_{n=1}^{N} \cos ^{2}\left[\omega_{n}^{\prime}(x, y)+\delta_{n}\right] & \sum_{n=1}^{N} \sin \left[\omega_{n}^{\prime}(x, y)+\delta_{n}\right] \\
\sum_{n=1}^{N} \sin \left[\omega_{n}^{\prime}(x, y)+\delta_{n}\right] & \sum_{n=1}^{N} \cos \left[\omega_{n}^{\prime}(x, y)+\delta_{n}\right] \times & \sum_{n=1}^{N} \sin ^{2}\left[\omega_{n}^{\prime}(x, y)+\delta_{n}\right]
\end{array}\right],}
\end{aligned}
$$

$$
\mathbf{X}(x, y)=\left[\begin{array}{lll}
a & B(x, y) & C(x, y)
\end{array}\right]^{T},
$$

$$
\mathbf{B}(x, y)=\left[\sum_{n=1}^{N} d_{n}^{m}(x, y) \quad \sum_{n=1}^{N} d_{n}^{m}(x, y) \times \quad \omega_{n}^{\prime}(x, y)+\delta_{n}\right] \quad \sum_{n=1}^{N} \sin \left[\omega_{n}^{m}(x, y)+\delta_{n}\right]^{T} .
$$

Form Eqs. (18)-(21) the unknowns $a, B(x, y)$ and $C(x, y)$ can be solved and $\Phi(x, y)$ can be determined: 


$$
\Phi(x, y)=\tan ^{-1}[-C(x, y) / B(x, y)]
$$

Step 2: Estimate $\delta_{n}$ by the least-squares method using $\Phi(x, y)$ estimated by Step 1 . For this purpose Eq. (15) can also be rewritten as:

$$
\begin{aligned}
& \tilde{d}_{n}(x, y)= \\
& a+B_{n}^{\prime} \cos \left[\omega_{n}^{\prime}(x, y)+\Phi(x, y)\right]+C_{n}^{\prime} \sin \left[\omega_{n}^{\prime}(x, y)+\Phi(x, y)\right]
\end{aligned}
$$

where $B_{n}^{\prime}=b \cos \delta_{n}$ and $C_{n}^{\prime}=-b \sin \delta_{n}$. The sum of the squares error in each frame is:

$$
S_{n}^{\prime}=\sum_{(x, y)}\left[\tilde{d}_{n}(x, y)-d_{n}^{m}(x, y)\right]^{2}
$$

The least-squares solution which minimizes $S_{n}^{\prime}$ is:

$$
\mathbf{X}_{n}^{\prime}=\mathbf{A}_{n}^{\prime-1} \mathbf{B}_{n}^{\prime}
$$

where

$$
\begin{aligned}
& \mathbf{A}_{n}^{\prime}= \\
& {\left[\begin{array}{ccc}
M & \sum_{x, y} \cos \left[\omega_{n}^{\prime}(x, y)+\Phi(x, y)\right] & \sum_{x, y} \sin \left[\omega_{n}^{\prime}(x, y)+\Phi(x, y)\right] \\
\sum_{x, y} \cos \left[\omega_{n}^{\prime}(x, y)+\Phi(x, y)\right] & \sum_{x, y} \cos ^{2}\left[\omega_{n}^{\prime}(x, y)+\Phi(x, y)\right] & \sum_{x, y} \sin \left[\omega_{n}^{\prime}(x, y)+\Phi(x, y)\right] \times \\
\sum_{x, y} \sin \left[\omega_{n}^{\prime}(x, y)+\Phi(x, y)\right] & \sum_{x, y} \cos \left[\omega_{n}^{\prime}(x, y)+\Phi(x, y)\right] \times & \left.\sum_{x, y}^{\prime}(x, y)+\Phi(x, y)\right] \\
\sin ^{2}\left[\omega_{n}^{\prime}(x, y)+\Phi(x, y)\right]
\end{array}\right]} \\
& \mathbf{X}_{n}^{\prime}=\left[\begin{array}{lll}
a & B_{n}^{\prime} & C_{n}^{\prime}
\end{array}\right]^{T}, \\
& \mathbf{B}_{n}^{\prime}=\left[\sum_{x, y} d_{n}^{m}(x, y) \quad \sum_{x, y} d_{n}^{m}(x, y) \times \quad \omega_{n}^{\prime}(x, y)+\Phi(x, y)\right] \quad \sum_{x, y}^{d_{n}^{m}(x, y) \times} \sin \left[\omega_{n}^{\prime}(x, y)+\Phi(x, y)\right]^{T} .
\end{aligned}
$$

Hence the unknowns $a, B_{n}^{\prime}$ and $C_{n}^{\prime}$ can be obtained by Eqs. (25)-(28). Then, $\delta_{n}$ can be calculated by:

$$
\delta_{n}=\tan ^{-1}\left[-C_{n}^{\prime} / B_{n}^{\prime}\right]
$$

The two steps described above are repeated in the way that $\delta_{n}$ estimated from Step 2 is employed in Step 1 to obtain a better estimation of $\Phi(x, y)$, and $\Phi(x, y)$ obtained in Step 1 is utilized in Step 2 to yield a better estimation of $\delta_{n}$. The iterative process will stop when the convergence condition is met:

$$
\left|\left(\delta_{n}^{k}-\delta_{1}^{k}\right)-\left(\delta_{n}^{k-1}-\delta_{1}^{k-1}\right)\right|<\varepsilon
$$

Then $\Phi(x, y)$ will be taken as the correct phase distribution. In the above $k$ is the number of iterations and $\varepsilon$ is the accuracy requirement, e.g., $10^{-4}$. In the experiments presented below, we found that a very good convergence can always be achieved, e.g., $k$ is around 10 when $\varepsilon=10^{-4}$.

Experiments have been carried out to verify the proposed method, where the object is the mask shown in Fig. 1(a) and the PSP is of 3-step. The object was moved on step-by-step basis in order to emulate the instantaneous positions of the object when the multiple image patterns are taken. Note that in practice, the shutter of the camera must be fast enough in order to avoid blurry in the captured images. As the camera in our laboratory is not fast enough, we kept the object static at each step when the fringe patterns are taken.

In order to obtain $\Phi(x, y)$ from Eq. $(15), \omega_{n}^{\prime}(x, y)$ should be available. To this end we should obtain $\left(\bar{r}_{11}, \bar{r}_{12}, \bar{r}_{21}, \bar{r}_{22}, \bar{t}_{1}, \bar{t}_{2}\right)$ in Eq. (13). Three marks are employed and placed on the object as shown in Fig. 1(a). The centers of the marks are extracted using the same approach described in Ref. [3], which are used as the corresponding points between multiple fringe patterns. As Eqs. (3) and (4) show that $\bar{t}_{3}$ is independent of $\left(\bar{r}_{11}, \bar{r}_{12}, \bar{r}_{21}, \bar{r}_{22}, \bar{t}_{1}, \bar{t}_{2}\right)$, the method described in Ref. [3] can be employed to obtain $\left(\bar{r}_{11}, \bar{r}_{12}, \bar{r}_{21}, \bar{r}_{22}, \bar{t}_{1}, \bar{t}_{2}\right)$. The following experiments have been conducted:

Experiment 1: We applied the traditional 3-step PSP to the mask when the mask is kept static during the measurement. The measurement result is shown in Fig. 1(e), which matches expectations of the traditional 3-step PSP.

Experiment 2: Then, we moved the object in the direction of height for $3 \mathrm{~mm}$ and $4 \mathrm{~mm}$ in the second and third step of PSP respectively. The result with traditional PSP is shown in Fig. 1(b) and there are obvious errors. In contrast, when the proposed algorithm is applied to the case where the object is moved by the same amount as above, significant improvement can be obtained as shown in Fig. 1(f).

Experiment 3: In the third experiment, the object is rotated clockwise in $x-y$ plane for $0.0599 \mathrm{rad}$ and moved in the direction of height for $5 \mathrm{~mm}$ in the second step of PSP; then, the object is rotated clockwise in $x-y$ plane for $0.0256 \mathrm{rad}$, and moved in the direction of height for $3 \mathrm{~mm}$ in the third step of PSP. The result with the traditional PSP is shown in Fig. 1(c) showing significant errors. With the proposed algorithm, the result is depicted in Fig. $1(\mathrm{~g})$, also showing significant improvement.

Experiment 4: For the fourth experiment, the object is moved $3 \mathrm{~mm}$ in $\mathrm{x}^{-}$-direction, $5 \mathrm{~mm}$ in $\mathrm{y}^{-}$ direction, $3 \mathrm{~mm}$ in the direction of height and rotated clockwise in $x-y$ plane for $0.0295 \mathrm{rad}$ in the second step of PSP; and then in the third step of PSP, the object is moved $2 \mathrm{~mm}$ in the $\mathrm{x}^{-}$direction, $4 \mathrm{~mm}$ in $\mathrm{y}^{-}$ direction, $2 \mathrm{~mm}$ in the direction of height and rotated clockwise for $0.0277 \mathrm{rad}$ in $x-y$ plane. Figure 1(d) shows the result with traditional PSP. Significant errors occur in the result. The result with the proposed algorithm is shown in Fig. 1(h), again demonstrating significant improvement.

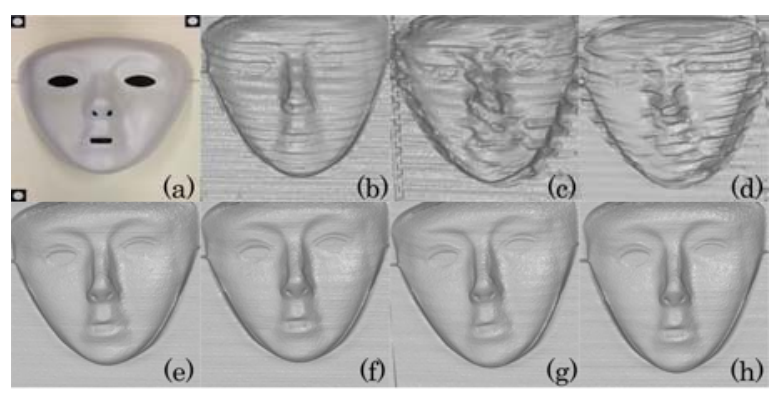

Fig. 1. Comparison of measurement results of traditional PSP and the method proposed. 
In order to evaluate the performance of the proposed algorithm, we also computed the RMS (root mean square) errors of the height for all the points on the object surface, the iterative times and the height offset of the proposed method for the above experiments. The results are shown in Table 1. As the exact shape of the mask is not available, the reconstruct result for the static object in Fig. 1(e) is used as the reference for our computation. Table 1 shows that the proposed method has significant accuracy improvement and fast convergence.

Table 1. Accuracy and iterative times $\left(\varepsilon=10^{-4}\right)$

\begin{tabular}{ccccc}
\hline $\begin{array}{c}\text { Experiment } \\
\text { number }\end{array}$ & $\begin{array}{c}\text { RMS errors } \\
\text { (traditional } \\
\text { PSP) }\end{array}$ & $\begin{array}{c}\text { RMS errors } \\
\text { (proposed } \\
\text { method) }\end{array}$ & $\begin{array}{c}\text { Iterative } \\
\text { times }\end{array}$ & $\begin{array}{c}\text { Height } \\
\text { offset }\end{array}$ \\
\hline $\begin{array}{c}\text { Experiment } \\
2\end{array}$ & $10.385 \mathrm{~mm}$ & $0.071 \mathrm{~mm}$ & 9 & $\begin{array}{c}0.002 \\
\mathrm{~mm}\end{array}$ \\
$\begin{array}{c}\text { Experiment } \\
3\end{array}$ & $62.946 \mathrm{~mm}$ & $0.089 \mathrm{~mm}$ & 12 & $\begin{array}{c}0.006 \\
\mathrm{~mm}\end{array}$ \\
$\begin{array}{c}\text { Experiment } \\
4\end{array}$ & $57.174 \mathrm{~mm}$ & $0.083 \mathrm{~mm}$ & 14 & $\begin{array}{c}0.004 \\
\mathrm{~mm}\end{array}$ \\
\hline
\end{tabular}

We also compared the accuracy of the results shown in Fig. 1(d) and Fig. 1(h). The absolute errors associated with these two cases are shown in Fig. 2. It can be seen that the absolute error in Fig. 2(b) using the proposed method is significant smaller than the error of the traditional PSP in Fig. 2(a).

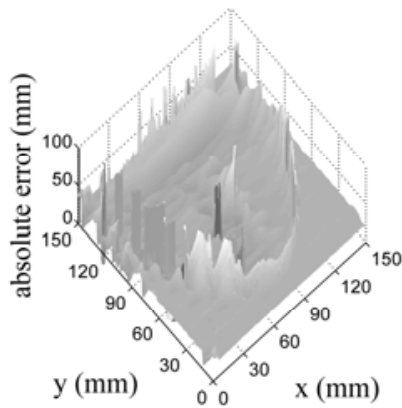

(a)

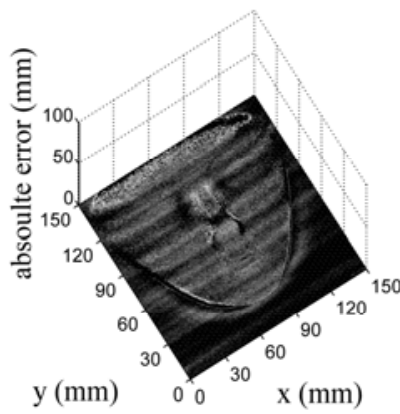

(b)
Fig. 2. (a)-(b) The absolute error for Fig. 1(d) and Fig. 1(h).

We also studied how the amount of movement impacts on the RMS error and the result is shown in Fig. 3. In the second step and third step of PSP, the object moves for the same distance and the movement distance in Fig. 3 is the sum of the movement of the two steps. For each movement, 8 experiments are carried out. Fig. 3 shows the mean of the RMS errors and the error bars giving the standard deviation for the 8 experiments at each movement distance. It is seen that RMS error slightly increases with the movement distance. This may be due to the influence of geometric structure of the system, as we only used the ideal model for the system structure, the camera and the projector. It is expected that the error can be reduced by means of accurate calibration of the system $[7,8]$.

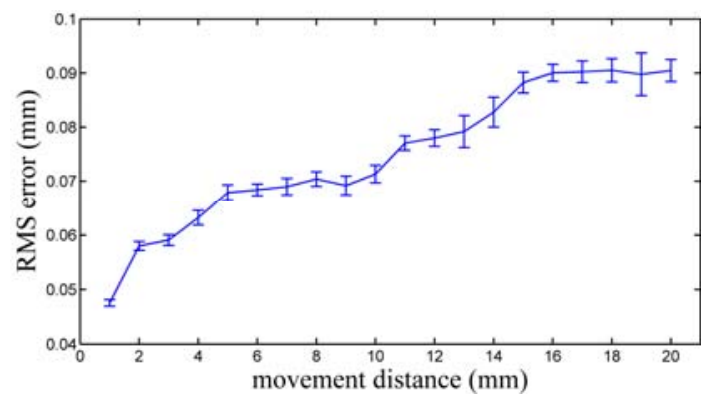

Fig. 3. The relationship between the accuracy of the proposed method and the movement distance in the direction of height.

The computation cost associated with the proposed method was also analyzed. Among other operations, the wrapped phase extraction in Eqs. (18)-(22) is most time consuming due to a matrix inversion for each pixel of the fringe patterns. However, parallel computing can be used as the computation can be independently implemented for each individual point. We employed such an approach using Matlab on a Dell Vostro 470 computer with $3.4 \mathrm{GHz}$ CPU and $8 \mathrm{G}$ memory, and it took 0.41 second to complete one iteration of Step 1 and Step 2 of the proposed algorithm for the fringe pattern of 500 by 500 pixels. It is expected that the time can be further reduced if dedicated hardware such as digital signal processors is employed [9].

In summary, a new approach has been proposed to determine the phase map of phase-shifted fringe patterns reflected from a rigid object subject to a $3-\mathrm{D}$ movement. Firstly, a new model is presented to describe the fringe patterns influenced by the $3-\mathrm{D}$ movement. Then, an iterative least-squares method is proposed, which is able to estimate the correct phase map without requiring the knowledge of the height variations. The estimated phase map can be used to reconstruct the $3-\mathrm{D}$ shape with improved accuracy performance in comparison to conventional PSP approach. Experimental results are also presented to verify the effectiveness of the proposed method.

\section{References}

[1] S. Zhang and S. T. Yau, Opt. Eng. 46, 113603 (2007).

[2] Y. Wang, S. Zhang and J. H. Oliver, Opt. Express 19, 8539 (2011).

[3] L. Lu, J. Xi, Y. Yu and Q. Guo, Opt. Express 21, 30610 (2013).

[4] Z. Wang and B. Han, Opt. Lett. 29, 1671 (2004).

[5] X. F. Xu, L. Z. Cai, X. F. Meng, G. Y. Dong and X. X. Shen, Opt. Lett. 31, 1966 (2006).

[6] C. Guo, B. Sha, Y. Xie and X. Zhang, Opt. Lett. 39, 813 (2014).

[7] Z. Zhang, IEEE Trans. Pattern Anal. Mach. Intell. 22, 1330 (2000).

[8] S. Zhang and P. S. Huang, Opt. Eng. 45, 083601 (2006).

[9] R. Juarez-Salazar, C. Robledo-Sanchez, F. GuerreroSanchez, and A. Rangel-Huerta, Opt. Express 22,4738 (2014). 
